\title{
Use of Volatile Anesthetics in Extracorporeal Circulation
}

\author{
Caetano Nigro Neto, TSA ${ }^{\text {, }}$ Maria Angela Tardelli, TSA ${ }^{2}$, Paulo Henrique Dagola Paulista ${ }^{3}$
}

\begin{abstract}
Nigro Neto C, Tardelli MA, Paulista PHD - Use of Volatile Anesthetics in Extracorporeal Circulation.
Background and objectives: The use of volatile anesthetics in cardiac surgery is not recent. Since the introduction of halothane in clinical practice, several cardiac surgery centers started to use these anesthetics constantly.

Content: In the last years a great number of studies have shown that the volatile anesthetics have a protecting effect against myocardial ischemic dysfunction. Experimental evidences have shown that the halogenated anesthetics have cardioprotective effects that cannot be only explained by coronary flow alterations or by the balance between myocardium available and consumed oxygen. In addition to that, the use of volatile anesthetics during extracorporeal circulation (ECC) in cardiac surgery plays an important role. Recent studies have proven that these agents have cardioprotective properties and produce better results when the volatile anesthetic is used during the whole surgery procedure, including ECC. The use of halogenated anesthetics through calibrated vaporizers adapted to the ECC circuit via oxygenator membranes has become popular. Therefore, the professionals involved such as anesthesiologists and perfusionists should learn specifcs in order to solve possible doubts.
\end{abstract}

Keywords: Anesthetics, Volatile; Cardiac Surgical Procedures; Extracorporeal Circulation; Ischemic Preconditioning; Oxygenators, Membrane.

\section{INTRODUCTION}

The use of volatile anesthetics in cardiac surgery is not recent. Since the introduction of halothane in clinical practice, several cardiac surgery centers had been constantly using these anesthetics ${ }^{1}$. However, volatile anesthetics were not used for a while due to studies that presented controversies concerning its use ${ }^{2,3}$. The decrease in the use of volatile agents can be explained by the beginning of total venous anesthesia administration in cardiac surgery with high doses of opioids, as it is considered a safe anesthetic technique of great cardiovascular stability ${ }^{4,5}$.

In the last years a great number of studies have shown that the volatile anesthetics have a protecting effect against myocardial ischemic dysfunction. Experimental evidences have shown that the halogenated agents have cardioprotective effects that cannot be only explained by coronary flow alterations or by the balance between the myocardium available and consumed oxygen. On the contrary, it seems that the volatile anesthetics have direct cardioprotective proper-

Received from the Instituto Dante Pazzanese de Cardiologia, Brazil.

1. PhD, Anesthesiology, Universidade Federal de São Paulo (UNIFESP); Associate Physician from the Clínica de Anestesia São Paulo

2. Professor, Anesthesia, UNIFESP; Head of the Discipline of Anesthesiology, Pain and Intensive Care Medicine, UNIFESP

3. Physician; Cardiac Surgeon, Fundação Adib Jatene

Submitted on March 6, 2011

Approved on August 3, 2011

Correspondence to:

Caetano Nigro, MD

Rua Peixoto Gomide, 502/173B

01409000 - São Paulo, SP, Brazil

Email: caenigro@uol.com.br ties ${ }^{6-8}$. Therefore, its use became popular again in cardiac surgery, especially with the introduction of safer agents from a cardiovascular perspective, such as isoflurane, sevoflurane and desflurane.

Some concepts, as ischemic and anesthetic pre- and postconditioning, play an important role in order to understand these protective effects.

\section{Ischemic pre- and post-conditioning}

Ischemia of a certain organ promotes tissue injury whose severity depends on the duration of the ischemic event and on the injuries due to subsequent reperfusion of the same area. The extent of the damage caused to this organ will determine the subsequent systemic alterations.

Organ pre-conditioning is related to the possible effect of the pretreatment with noxious stimulus, in which cellular tolerance to subsequent noxious stimulus increases. When the harmful effect is an ischemic event, it is called an ischemic pre-conditioning. In the myocardium, ischemic pre-conditioning would represent an adaptive endogenous response provoked by short episodes of cellular ischemia, which would lead to a greater and intrinsic protection of the myocardium cell against a subsequent episode of a more prolonged ischemia ${ }^{9}$. In the last few years, scientists have put a lot of effort in research into the cellular mechanism that really explains the heart ischemic pre-conditioning ${ }^{10,11}$. Concerning duration, the protective effects are limited and they are manifested in two phases: an initial phase occurring immediately after the noxious stimulus that induces strong protection for a short 
period of time (between 1 and 2 hours); and a delayed one, which occurs approximately 24 hours after the initial stimulus, even without another noxious stimulus. This phase induces a less intense protection during a longer period (about three days) ${ }^{8,12}$.

Most of experimental findings indicate that the preservation of mitochondrial function, which is due to the activation of potassium ATP-dependent channels, is vital for the cardioprotective effects against ischemia, as it reduces intracellular calcium overload, preserves energy reserves more efficiently and prevents the activation of the effects that cause apoptosis and cellular necrosis. The delayed phase of pre-conditioning depends on the cellular memory formation, which is attributed to the synthesis or activation of proteins that present a cytoprotection effect, such as the induction of several antioxidant enzymes or the synthesis of proteins involved in cell stabilization ${ }^{13}$.

In the ischemic post-conditioning, short ischemia episodes would be applied in the initial phase of reperfusion, therefore reducing the extent of the cell injuries that usually occur during this period.

Other experimental studies have shown that ischemic cardiac pre-conditioning can be extinguished or limited by the use of pharmacological agents that block or stimulate certain phases of the intracellular events series ${ }^{13-16}$. From a clinical point of view, cardiac pre-conditioning with pharmacological agents is preferred rather than the ischemic pre-conditioning, due to the risk of exposure of an ischemic myocardium to a transitory ischemia.

\section{Anesthetic pre- and post-conditioning}

When the pharmacological agents that present the pre- and post-conditioning are anesthetic agents, the effects are called anesthetic pre- and post-conditioning.

Among these agents, several studies show that the halogenated anesthetics are capable of promoting post-conditioning directly, or increasing indirectly the ischemic pre- and postconditioning against ischemia and reperfusion injuries 12,14,17. This mechanism is not totally clear yet, but is seems to mimic ischemic pre-conditioning.

Initially, it was believed that the halogenated agents protection in the heart was an indirect effect, due to contraction and oxygen consumption decrease. Nowadays it is quite evident that the effect is actually direct, and it involves the activation of various intracellular pathways that will promote protection against dysfunction and myocardial necrosis. Both the ischemic and the anesthetic pre-conditioning in the heart present the same cellular mechanism, which involves the activation of the mitochondrial and sarcolemma $\mathrm{K}_{\mathrm{ATP}}$-dependent channels, resulting in overload decrease in the mitochondrial and myoplasmatic calcium 7,17-19. Additionally, studies have shown that the halogenated anesthetics promote protection against ischemia-reperfusion injuries by pre-conditioning blood vessels, protecting against vascular injuries and impeding the endothelium contribution to the pro-inflammatory and thrombo- genic events related to ischemia-reperfusion injuries ${ }^{20}$. In this case, the halogenated anesthetics inhibit the expression of the molecules responsible for activating leukocyte adhesion and transmigration, and improve the vascular reactivity, preserving the capacity of vasodilation by $\mathrm{K}_{\text {ATP }}$ channels activation. They reduce intracellular calcium in the vascular smooth muscle and release nitric oxide 17,21,22.

\section{Volatile anesthetics and cardiac surgery}

The use of halogenated anesthetics in clinical practice has played an important role in the prevention and treatment of intraoperative cardiac ischemic dysfunction, due to recent discoveries regarding their cardioprotective properties. Considering that the protection of halogenated anesthetics against ischemia-reperfusion injuries is the result of a direct effect and the potentialization of pre-conditioning ischemic effects, most studies regarding halogenated anesthetics include patients showing myocardial ischemia. These would be the patients to have a better benefit from the effects of cardioprotective anesthetics ${ }^{12,23-25}$.

Clinical implications of cardioprotective volatile anesthetics during anesthesia are hemodynamic function better preservation and reduced release of biochemical markers of myocardial injury. Two studies assessed the effects of volatile anesthetics administered during the whole operative period of patients undergoing myocardial revascularization, including the ECC period. Patients who received volatile anesthesia, in comparison to total intravenous anesthesia, presented a better preservation of the cardiac function after the extracorporeal circulation and released smaller quantities of cardiac troponin I into blood ${ }^{23,26}$. Other studies reported the use of different types of markers, such as creatine kinase MB, tumor necrosis factor alpha, and total and brain natriuretic peptide. The results also indicated that the patients who were administered halogenated anesthetics presented a lower increase of these markers ${ }^{27,28}$. Better renal function was also observed, but the study protocol does not allow us to say if this effect is due to better cardiac output or because of a direct protective action of the halogenated anesthetic on the kidney ${ }^{28}$.

The impact of other consequences of this protective effect, such as the length reduction in the intensive care units, and the decrease of postoperative morbidity and mortality have inspired many new publications. Some studies indicate that patients receiving volatile anesthetics stay less time in intensive care units and are discharged earlier than those who receive total intravenous anesthesia $27,29,30$. A recent study observed that a 10-minute administration of sevoflurane in the beginning of ECC before aortic clamping can be associated to a lower rate of cardiovascular complications in the first postoperative year ${ }^{22}$. According to studies, the volatile anesthetics use in cardiac surgery decreases perioperative morbidity and can also reduce mortality ${ }^{31}$. 


\section{Extracorporeal circulation and volatile anesthetics}

Volatile anesthetics during ECC in cardiac surgeries are increasing. A recent research has proven that these agents have cardioprotective properties, and there is a better result when the volatile anesthetic is used during the whole surgical procedure, including ECC ${ }^{24}$.

Several studies that assessed patients undergoing coronary arterial bypass surgery have approached the clinical possible implications of these cardioprotective effects in the postoperative myocardial function. Most of these studies involved a pre-conditioning protocol in which the volatile anesthetic was administered before myocardial ischemia $28,32,33$. Although most of these studies had reported some cardioprotective effects, the magnitude and extent of this protection varied. Another research group assessed the effects of volatile anesthetics administered throughout the procedure ${ }^{26}$. The discrepancies concerning the relevance of cardioprotection should be related to the duration and to the moment of administration of these volatile anesthetics as contributors to the myocardial protection. One of the most engaged authors in this research is De Hert et al. ${ }^{24}$ According to Schlack et al. ${ }^{20}$, it is currently not possible to answer about the dose and time optimization of volatile anesthetic application. However, administration during the whole procedure, including the ECC through the membrane oxygenator seems to be the most appropriate one ${ }^{34}$.

It is important to mention safety issues concerning the use of volatile anesthetics during ECC. The use of these agents through calibrated vaporizers adapted to the ECC circuit via the oxygenator membranes has become popular, even though it is not a new technique. Therefore, the professionals involved, such as anesthesiologists and perfusionists, should learn specifics in order to discuss possible doubts.

The presence of anesthetic vaporizers in the ECC circuit should involve new strategies in order to improve patient safety.

Aware of the changes in anesthetic techniques during ECC, some companies responsible for manufacturing oxygenators already present their oxygenators with safety certification for use of volatile anesthetic agents in clinical practice. Besides, some ECC circuits already have calibrated vaporizers for volatile anesthetics attached to the ECC machines (Figure 1).

The anesthetic agent vaporization in the ECC is a process involving a calibrated vaporizer attached to the circuit, behind the blender responsible for the fresh gases release (oxygen and compressed air) (Figure 2). Fresh gas enters the vaporizer and, as the desired concentration is regulated, the volatile anesthetic is blended with the fresh gas flow which is vaporized in the circuit until the membrane oxygenator (Figures 3 and 4).

The membranes of the oxygenators available in Brazil used in the surgeries with ECC have the same basic raw material component when manufactured - the polypropylene. However, they differ in the oxygenator's external shape, and in the internal organization of the membrane, additionally to other components of the ECC equipment.

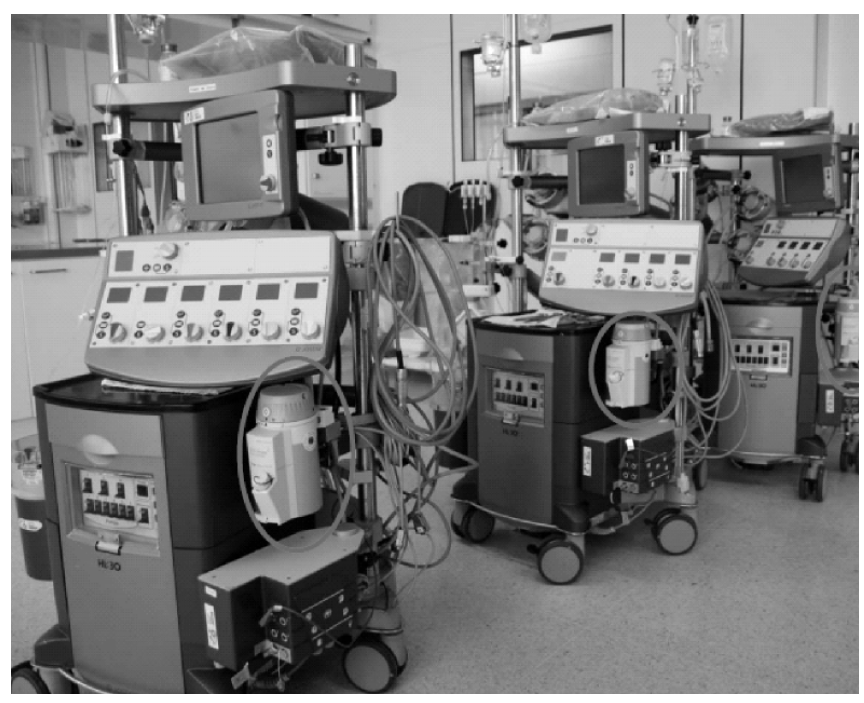

Figure 1 - Vaporizers Calibrated for Sevoflurane Attached to the Extracorporeal Circulation Circuit by the Manufacturer.

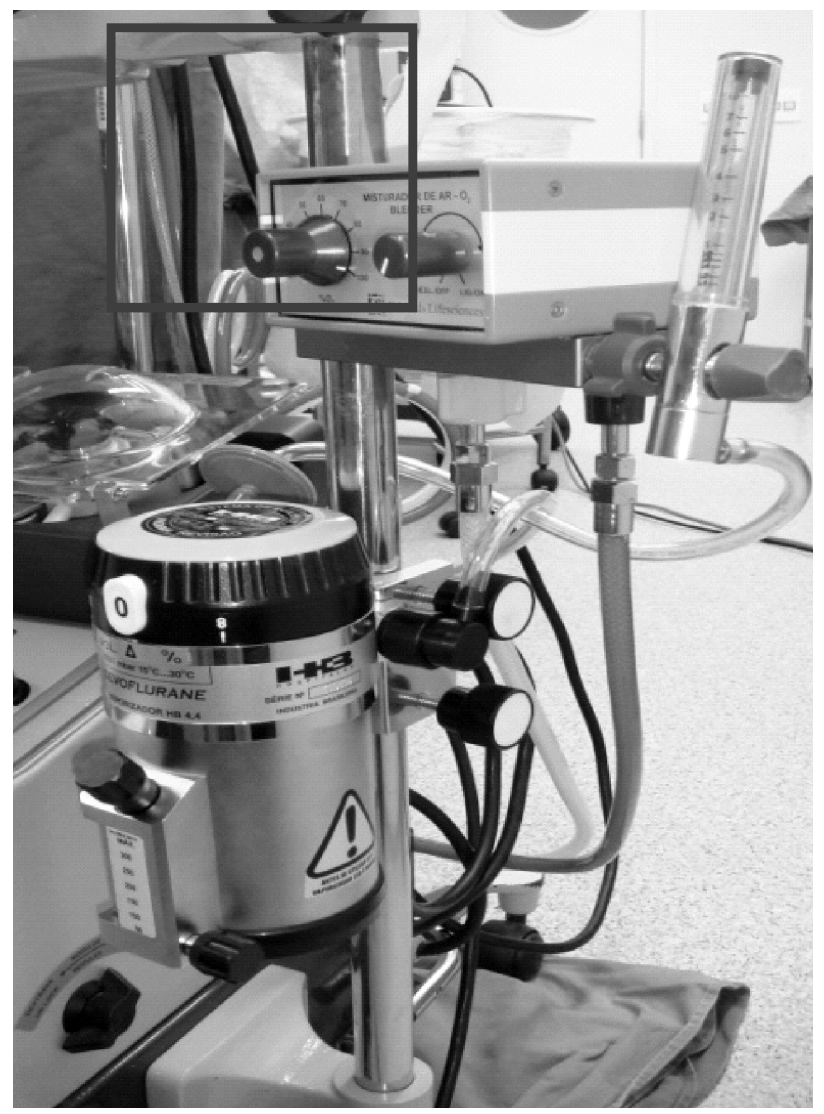

Figure 2 - Oxygen and Compressed Air Blender.

Other membranes qualified as diffusion and made of silicon are used in circuits to assist postoperative mechanical circulatory support, such as the ECMO (extracorporeal membrane oxygenation) ${ }^{35,36}$. Although these membranes have longer service life, they are not compatible with volatile anesthetics use, as described by Matthias ${ }^{34}$. 


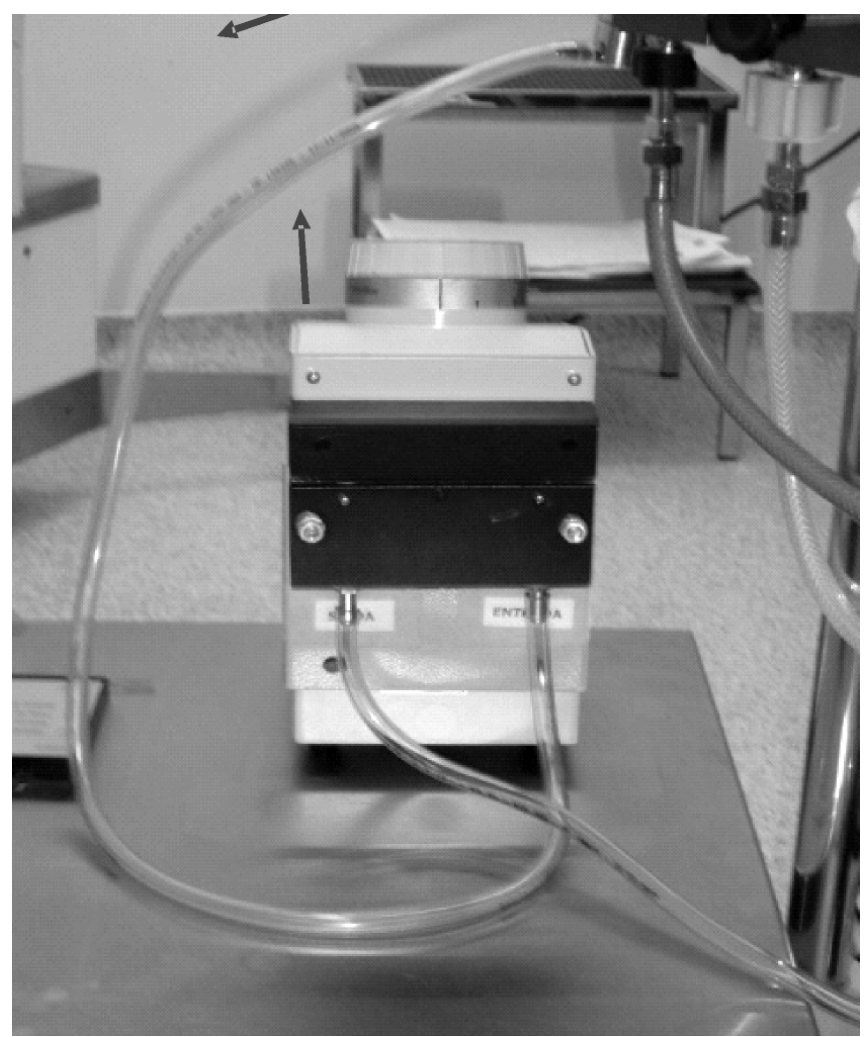

Figure 3 - Direction of the Fresh Gases from Blenders and Entry to Vaporizer.

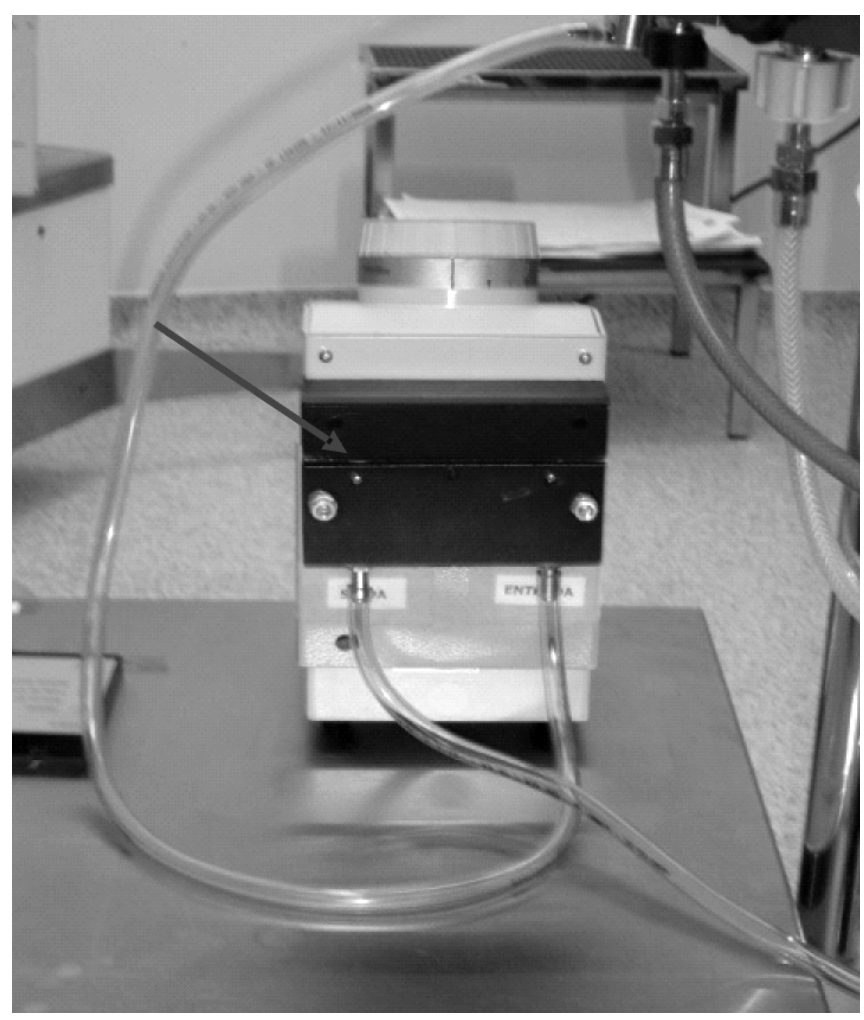

Figure 4 - Fresh Gas Output Together with Vaporized Volatile Anesthetic towards the Membrane Oxygenator.

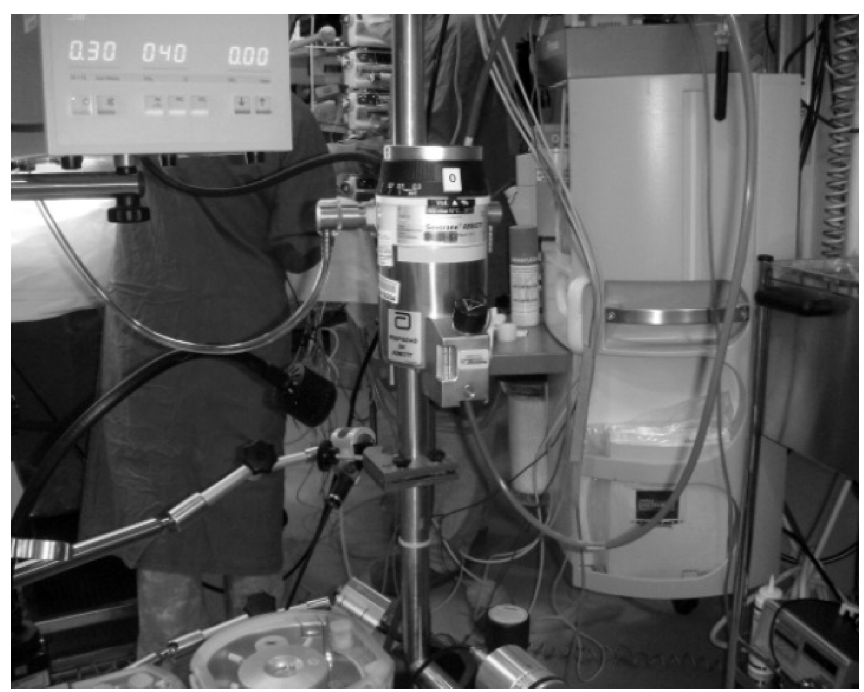

Figure 5 - Vaporizer Adapted to the ECC Circuit.

\section{The experience of the Instituto Dante Pazzanese de Cardiologia}

In the institute where this study was carried out, vaporized sevoflurane was used in the ECC circuit in patients undergoing several types of cardiac surgeries, such as myocardial revascularization, valve replacement and correction of congenital cardiopathy.

As ECC machine with attached vaporizers are not available at the institute, a vaporizer calibrated for sevoflurane is adapted to the ECC circuit (Figures 3, 4 and 5). It is important to mention that, whenever possible, the gas rate in the oxygenator should be monitored. In addition, it is necessary to adapt a system to depollute the operating room, as the excess of gases administrated in the oxygenators are directly released into the operating room.

In order to change the concentration of sevoflurane in ECC, it is necessary to consider the mean arterial pressure values, and, more importantly, the blood flow during perfusion, as well as the values of bispectral index (BIS). The maximum inspired concentration of sevoflurane is $3 \%$. Therefore, satisfactory results have been achieved during the entire perfusion. Physicians are using less vasodilator agents (sodium nitroprusside) and are controlling the appropriate values of BIS, without using additional doses of hypnotics during the entire ECC.

\section{CONCLUSIONS}

Considering that the effectiveness of myocardial protection produced by the halogenated anesthetics in cardiac surgery has already been proved and that it is highly beneficial when it is administered during the whole surgery, including ECC, this technique has become more popular in all cardiac surgery centers. When the anesthesiologists become confident on using vaporizers attached to the ECC system, it will be possible to use an anesthetic technique capable of not only improving patient transport during surgery, but also contributing in the short and long terms to the decrease of postoperative morbidity and mortality. 
13. Das B, Sarkar C - Pharmacological preconditioning by levosimendan is mediated by inducible nitric oxide synthase and mitochondrial KATP channel activation in the in vivo anesthetized rabbit heart model. Vascul Pharmacol, 2007;47(4):248-256.

14. Lai IR, Chang KJ, Tsai HW, Chen CF - Pharmacological preconditioning with simvastatin protects liver from ischemia-reperfusion injury by heme oxygenase-1 induction. Transplantation, 2008;85(5):732738.

15. Parratt JR - Protection of the heart by ischaemic preconditioning: mechanisms and possibilities for pharmacological exploitation. Trends Pharmacol Sci, 1994;15(1):19-25.

16. Toombs CF, Moore TL, Shebuski RJ - Limitation of infarct size in the rabbit by ischaemic preconditioning is reversible with glibenclamide. Cardiovasc Res, 1993;27(4):617-622.

17. Tanaka K, Ludwig LM, Krolikowski JG et al. - Isoflurane produces delayed preconditioning against myocardial ischemia and reperfusion injury: role of cyclooxygenase-2. Anesthesiology, 2004;100(3):525531.

18. Lim YJ, Zheng S, Zuo Z - Morphine preconditions Purkinje cells against cell death under in vitro simulated ischemia-reperfusion conditions. Anesthesiology, 2004;100(3):562-568.

19. Zhang $Y$, Irwin MG, Wong TM - Remifentanil preconditioning protects against ischemic injury in the intact rat heart. Anesthesiology, 2004;101(4):918-923.

20. Schlack W, Preckel B, Stunneck D, Thamer V - Effects of halothane, enflurane, isoflurane, sevoflurane and desflurane on myocardial reperfusion injury in the isolated rat heart. $\mathrm{Br} \mathrm{J}$ Anaesth, 1998;81(6):913919.

\section{REFERÊNCIAS/REFERENCES}

1. Pederson $\mathrm{D}$, Secher $\mathrm{O}-$ Halothane anaesthesia in cardiac surgery. Acta Anaesthesiol Scand, 1958;2(4):169-179.

2. Karliczek G, Hempelmann G, Piepenbrock S - A comparison of the cardiovascular effects of enflurane, halothane, methoxyflurane and fluroxene during open cardiac surgery. Acta Anaesthesiol Belg, 1975;26(2-3):81-93.

3. Luk HN, Lin $\mathrm{Cl}$, Wei J, Chang $\mathrm{CL}$ - Depressant effects of isoflurane and halothane on isolated human atrial fibers. Anesthesiology, 1988;69(5):667-676.

4. Lunn JK, Stanley TH, Eisele J, Webster L, Woodward A - High dose fentanyl anesthesia for coronary artery surgery: plasma fentanyl concentrations and influence of nitrous oxide on cardiovascular responses. Anesth Analg, 1979;58(5):390-395.

5. Stanley TH, Webster LR - Anesthetic requirements and cardiovascular effects of fentanyl-oxygen and fentanyl-diazepam-oxygen anesthesia in man. Anesth Analg, 1978;57(4):411-416.

6. Kersten JR, Lowe D, Hettrick DA, Pagel PS, Gross GJ, Warltier DC - Glyburide, a KATP channel antagonist, attenuates the cardioprotective effects of isoflurane in stunned myocardium. Anesth Analg, 1996;83(1):27-33.

7. Preckel B, Schlack W, Comfere T, Obal D, Barthel H, Thamer V - Effects of enflurane, isoflurane, sevoflurane and desflurane on reperfusion injury after regional myocardial ischaemia in the rabbit heart in vivo. $\mathrm{Br} \mathrm{J}$ Anaesth, 1998;81(6):905-912.

8. Warltier DC, al-Wathiqui MH, Kampine JP, Schmeling WT - Recovery of contractile function of stunned myocardium in chronically instrumented dogs is enhanced by halothane or isoflurane. Anesthesiology, 1988;69(4):552-565.

9. Murry CE, Jennings RB, Reimer KA - Preconditioning with ischemia: a delay of lethal cell injury in ischemic myocardium. Circulation, 1986;74(5):1124-1136.

10. Auchampach JA, Grover GJ, Gross GJ - Blockade of ischaemic preconditioning in dogs by the novel ATP dependent potassium channel antagonist sodium 5-hydroxydecanoate. Cardiovasc Res, 1992;26(11):1054-1062.

11. Yao Z, Gross GJ - A comparison of adenosine-induced cardioprotection and ischemic preconditioning in dogs. Efficacy, time course, and role of KATP channels. Circulation, 1994;89(3):1229-1236.

12. De Hert SG - Cardioprotection with volatile anesthetics: clinical relevance. Curr Opin Anaesthesiol, 2004;17(1):57-62.
21. Collard CD, Gelman S - Pathophysiology, clinical manifestations, and prevention of ischemia-reperfusion injury. Anesthesiology, 2001;94(6):1133-1138.

22. Garcia C, Julier K, Bestmann L et al. - Preconditioning with sevoflurane decreases PECAM-1 expression and improves one-year cardiovascular outcome in coronary artery bypass graft surgery. $\mathrm{Br} \mathrm{J}$ Anaesth, 2005;94(2):159-165.

23. De Hert SG, ten Broecke PW, Mertens E et al. - Sevoflurane but not propofol preserves myocardial function in coronary surgery patients. Anesthesiology, 2002;97(1):42-49.

24. De Hert SG, Van der Linden PJ, Cromheecke S et al. - Cardioprotective properties of sevoflurane in patients undergoing coronary surgery with cardiopulmonary bypass are related to the modalities of its administration. Anesthesiology, 2004;101(2):299-310.

25. Landoni G, Fochi O, Torri G - Cardiac protection by volatile anaesthe-

26. De Hert SG, Cromheecke S, ten Broecke PW et al. - Effects of propofol, desflurane, and sevoflurane on recovery of myocardial function after coronary surgery in elderly high-risk patients. Anesthesiology, 2003;99(2):314-323.

27. El A, Rosseel PM, De Lange JJ et al. - Effect of sevoflurane on the ex vivo secretion of TNF-alpha during and after coronary artery bypass surgery. Eur J Anaesthesiol, 2003;20(5):380-384.

28. Julier K, da Silva R, Garcia C et al. - Preconditioning by sevoflurane decreases biochemical markers for myocardial and renal dysfunction in coronary artery bypass graft surgery: a double-blinded, placebocontrolled, multicenter study. Anesthesiology, 2003;98(6):1315-1327.

29. De Hert SG, Van der Linden PJ, Cromheecke S et al. renal dysfunction in coronary artery bypass graft surgery: a double-blinded, placebo- Choice of primary anesthetic regimen can influence intensive care unit length of stay after coronary surgery with cardiopulmonary bypass. Anesthesiology, 2004;101(1):9-20.

30. Guarracino F, Landoni G, Tritapepe L et al. renal dysfunction in coronary artery bypass graft surgery: a double-blinded, placebo- Myocardial damage prevented by volatile anesthetics: a multicenter randomized controlled study. J Cardiothorac Vasc Anesth, 2006;20(4):477-483.

31. Landoni G, Biondi-Zoccai GG, Zangrillo $A$ et al. - Desflurane and sevoflurane in cardiac surgery: a meta-analysis of randomized clinical trials. J Cardiothorac Vasc Anesth, 2007;21(4):502-511.

32. Haroun-Bizri S, Khoury SS, Chehab IR, Kassas CM, Baraka A - Does isoflurane optimize myocardial protection during cardiopulmonary bypass? J Cardiothorac Vasc Anesth, 2001;15(4):418-421. tics: a review. Curr Vasc Pharmacol, 2008;6(2):108-111. 
33. Penta de PA, Polisca P, Tomai F et al. - Recovery of LV contractility in man is enhanced by preischemic administration of enflurane. Ann Thorac Surg, 1999;68(1):112-118.

34. Matthias A - Volatile Anästhetika an der Herz Lungen Maschine Praktische Erfahrungen. Kardiotechnik. 2008;(1):14-17.

35. Walter EM, Meskishvili V, Huebler M et al. - Extracorporeal membrane oxygenation for intraoperative cardiac support in children with congenital heart disease. Interact Cardiovasc Thorac Surg, 2010;10(5):753-758.

36. Scherer M, Sirat AS, Moritz A, Martens S - Extracorporeal membrane oxygenation as perioperative right ventricular support in patients with biventricular failure undergoing left ventricular assist device implantation. Eur J Cardiothorac Surg, 2010;39:939-944. 